\title{
Firm spin and parity assignments for high-lying, low-spin levels in stable Si isotopes
}

\author{
J. Sinclair ${ }^{1,2}$, M. Scheck ${ }^{1,2, a}$, S. W. Finch ${ }^{3,4}$, Krishichayan ${ }^{3,4}$, U. Friman-Gayer ${ }^{5}$, W. Tornow ${ }^{3,4}$, G. Battaglia ${ }^{2,6}$, \\ T. Beck ${ }^{5}$, R. Chapman ${ }^{1,2}$, M. M. R. Chishti ${ }^{1,2}$, Ch. Fransen ${ }^{7}$, R. Gonzales ${ }^{4,8}$, E. Hoemann ${ }^{7}$, J. Isaak ${ }^{5}$, \\ R. V. F. Janssens ${ }^{4,8}$, D. A. Jaroszynski ${ }^{2,6}$, S. Johnson ${ }^{4,8}$, M. D. Jones ${ }^{4,8}$, J. M. Keatings ${ }^{1,2}$, N. Kelly ${ }^{1,2}$, J. Kleemann ${ }^{5}$, \\ D. Little ${ }^{4,8}$, B. Löher ${ }^{9}$, K. R. Mashtakov ${ }^{1,2}$, M. Müscher ${ }^{7}$, D. O’Donnell ${ }^{1,2}$, O. Papst ${ }^{5}$, E. E. Peters ${ }^{10}$, D. Savran ${ }^{9}$, \\ M. Schilling ${ }^{5}$, R. Schwengner ${ }^{11}$, P. Spagnoletti ${ }^{1,2}$, M. Spieker ${ }^{12,13}$, V. Werner ${ }^{5}$, J. Wilhelmy ${ }^{7}$, O. Wieland $^{14}$, \\ S. W. Yates ${ }^{10}$, A. Zilges ${ }^{7}$ \\ ${ }^{1}$ School of Computing, Engineering, and Physical Sciences, University of the West of Scotland, Paisley PA1 2BE, UK \\ 2 SUPA, Scottish Universities Physics Alliance, Edinburgh, UK \\ ${ }^{3}$ Department of Physics, Duke University, Durham, NC 27708-0308, USA \\ 4 Triangle Universities Nuclear Laboratory, Durham, NC 27708-0308, USA \\ ${ }^{5}$ Institut für Kernphysik, Technische Universität Darmstadt, 64289 Darmstadt, Germany \\ ${ }^{6}$ Department of Physics, University of Strathclyde, Glasgow G4 0NG, UK \\ ${ }^{7}$ Institut für Kernphysik, Universität zu Köln, 50937 Cologne, Germany \\ ${ }^{8}$ Department of Physics and Astronomy, University of North Carolina at Chapel Hill, Chapel Hill, NC 27599, USA \\ ${ }^{9}$ GSI Helmholtzzentrum für Schwerionenforschung GmbH, 64291 Darmstadt, Germany \\ ${ }^{10}$ Departments of Chemistry and Physics \& Astronomy, University of Kentucky, Lexington, KY 40506-0055, USA \\ ${ }^{11}$ Helmholtz-Zentrum Dresden-Rossendorf, 01328 Dresden, Germany \\ 12 NSCL, Michigan State University, East Lansing, MI 48824, USA \\ ${ }^{13}$ Department of Physics, Florida State University, Tallahassee, FL 32306, USA \\ ${ }^{14}$ INFN, Sezione di Milano, 20133 Milan, Italy
}

Received: 22 November 2019 / Accepted: 24 February 2020 / Published online: 6 April 2020

(C) The Author(s) 2020

Communicated by Calin Alexandru Ur

\begin{abstract}
A natural silicon target was investigated in a ${ }^{\text {nat }} \operatorname{Si}\left(\boldsymbol{\gamma}, \gamma^{\prime}\right)$ photon-scattering experiment with fully linearlypolarised, quasi-monochromatic $\boldsymbol{\gamma}$ rays in the entrance channel. The mean photon energies used were $\left\langle E_{\gamma}\right\rangle=9.33,9.77$, $10.17,10.55,10.93$, and $11.37 \mathrm{MeV}$, and the relative energy spread (full width at half maximum) of the incident beam was $\Delta E_{\gamma} /\left\langle E_{\gamma}\right\rangle \approx 3.5-4 \%$. The observed angular distributions for the ground-state decay allow firm spin and parity assignments for several levels of the stable even-even silicon isotopes.
\end{abstract}

\section{Introduction}

For a massive star to end its lifecycle in a type-II core collapse supernova, modern three-dimensional simulations have demonstrated the necessity for including neutrino-nucleus interactions as an additional heating mechanism (see, e.g., Refs. [1,2]). These interactions are for an even-even nucleus dominated by $1^{+}$levels $[3,4]$ forming Gamow-Teller (GT)

a e-mail: marcus.scheck@uws.ac.uk (corresponding author) resonances [5]. Considering that a massive star in the last weeks of its lifecycle fuses lighter nuclei to form ${ }^{28} \mathrm{Si}$, which subsequently acts as a seed to synthesise in the very last stage elements up to ${ }^{56} \mathrm{Ni} /{ }^{56} \mathrm{Fe}$, it can be safely assumed that there is a considerable amount of silicon, in particular ${ }^{28} \mathrm{Si}$, in the collapsing iron-nickel core or the shell surrounding it. Consequently, these Si nuclei are among the first to be exposed to the immense neutrino flux produced by the gravity-induced electron capture reactions, which trigger the core collapse. Therefore, it can be expected that the interaction of neutrinos with silicon nuclei contributes to the heating process. Of course, the temperatures $(T)$ of nuclei involved in a type-II supernova is far higher than the temperature of nuclei studied in the laboratory. The enhanced temperature causes a redistribution of the nucleons, which results in a thermal unblocking of otherwise fully occupied levels. This unblocking allows otherwise Pauli-forbidden Gamow-Teller strength for levels below the $T=0$ Fermi level to occur; e.g., see Refs. [6,7] and references therein. Nevertheless, establishing a clear picture of the GT resonances under laboratory conditions presents nuclear theory with a low-temperature 
test case. One necessity when establishing the experimental picture is a firm assignment of spins and parities to the possible $J^{\pi}=1^{+}$levels that form the GT resonances.

In the past, the stable silicon isotopes, in particular ${ }^{28} \mathrm{Si}$, were the subject of many studies [8-10]. Of relevance for this work are the results of previous ${ }^{28} \mathrm{Si}\left(e, e^{\prime}\right)[11],{ }^{28} \mathrm{Si}\left(p, p^{\prime}\right)$ [12], and ${ }^{28,30} \mathrm{Si}\left(\gamma, \gamma^{\prime}\right)$ [13] studies. The latter work used partially polarised bremsstrahlung, which allows firm spin and parity information to be extracted for strongly excited levels [14]. Interestingly, while both $\left(e, e^{\prime}\right)$ and $\left(\gamma, \gamma^{\prime}\right)$ data sets report firm spin- and parity $J^{\pi}$ information for several $1^{+}$levels, many of the $J^{\pi}$ values in the evaluated database [8] are given in parentheses. For ${ }^{30} \mathrm{Si}$, the experimental information given in Ref. [13] is currently not present in the database [10]. The proton-scattering experiments [12] confirmed the isovector nature for several of the observed $1^{+}$levels of ${ }^{28} \mathrm{Si}$. Furthermore, in spite of being observed in Ref. [13], no scattering cross sections for the $J^{\pi}=11^{-}$levels in ${ }^{28} \mathrm{Si}$ and ${ }^{30} \mathrm{Si}$ are given. For ${ }^{28} \mathrm{Si}$, these studies have shown that the isovector $M 1$ response is dominated by an extremely strongly excited $1^{+}$level at $11446 \mathrm{keV}$. For ${ }^{29} \mathrm{Si}$, no dedicated photonscattering experiment has been reported [9].

As in Ref. [15], the first objective of the photon-scattering experiments presented in this work was to exploit the wellknown excitation energy of the 11446-keV level as an energy calibration point for other nuclear resonance fluorescence (NRF) measurements. However, the opportunity to measure more energy settings in combination with the high sensitivity of the experimental setup allowed for an unambiguous assignment or confirmation of spin and parity combinations for excited levels in ${ }^{28} \mathrm{Si}$ and ${ }^{30} \mathrm{Si}$.

\section{Experiment}

The experiment was part of a NRF campaign at the HighIntensity $\gamma$-ray Source $(\mathrm{HI} \gamma \mathrm{S})$ at the Duke Free Electron Laser Laboratory [17] of the Triangle Universities Nuclear Laboratory (TUNL). At $\mathrm{HI} \gamma \mathrm{S}$, electrons are circulated in a storage ring, and emit, in a Wiggler section, free-electronlaser light in the (near-)visible part of the electromagnetic spectrum. After being reflected by an optical mirror, the laser light collides at a well-defined interaction point with a second bunch of high-energy electrons. In this process, two effects play a crucial role. First, Compton scattering preserves the well-defined polarisation of the free-electronlaser light and, second, the Lorentz transformation into the rest frame of the (in the laboratory frame highly relativistic) electrons boosts the energy of the Compton-backscattered photons (CBP) into the $\mathrm{MeV}$ regime. Basically, the energy of the photons from the free-electron laser and the Lorentz boost can be adjusted via the electron energy. A narrow angle acceptance for the CBPs results in a fully linearly- polarised, quasi-monochromatic photon beam. In the present experiment, the angle selection was done using a collimator ( $12.8 \mathrm{~mm}$ diameter), which was $\approx 57 \mathrm{~m}$ downstream from the collision point. The photon beam was adjusted to mean energies, $\left\langle E_{\gamma}\right\rangle$, of $9.33,9.77,10.17,10.55,10.93$, and $11.37 \mathrm{MeV}$. The energy spread corresponding to the full width at half maximum of a Gaussian-like distribution, $\Delta E_{\gamma}$, is $3.5-4 \%$ of the mean energy of the distribution with the above collimator. The measurement time was approximately $4 \mathrm{~h}$ for each energy setting.

Due to the limited momentum transfer, the absorption process for real photons is very spin selective and strongly favors dipole excitations. Hence, for even-even nuclei, $J^{\pi}=1^{\pi}$ levels are excited almost exclusively. E2-excited $2^{+}$levels are usually weakly populated and, often, their decay $\gamma$ rays cannot be resolved from the background caused by incomplete detector response, pile-up events, a few events from cosmic rays, and Compton-scattered photons. Using the direction of the incident photon beam as the quantisation axis (polar angle $\theta$ ), the angular distribution of the emitted $\gamma$ rays contains information about the angular momentum, $J$, of the excited level. Using fully linearly-polarised $\gamma$ rays in the entrance channel allows an azimuthal angle, $\phi$, with respect to the polarisation vector to be defined. Consequently, the momentum vector of the incident photon and its polarisation vector define the polarisation plane. In particular, the resonant cascades $0_{g s}^{+} \rightarrow J^{\pi} \rightarrow 0_{g s}^{+}$with excitation from and decay to the $0_{g s}^{+}$ground state exhibit a pronounced angular distribution, $W\left(J^{\pi}, \theta, \phi\right)$ [18]. In the phase convention of Krane, Steffen, and Wheeler [19], the angular distributions for an even-even nucleus are given as:

$$
\begin{aligned}
W\left(1^{\pi}, \theta, \phi\right)= & \frac{3}{4}\left[1+\cos ^{2} \theta+\pi \cdot \cos (2 \phi) \sin ^{2} \theta\right] \\
W\left(2^{+}, \theta, \phi\right)= & \frac{5}{8}[2+\cos (2 \theta)+\cos (4 \theta) \\
& \left.-(1+2 \cos (2 \theta)) \cdot 2 \cos (2 \phi) \cdot \sin ^{2} \theta\right] .
\end{aligned}
$$

Illustrations of these angular distributions can, for example, be found in Refs. [20,21]. For given spin and parity values, the angular distribution vanishes for specific angle combinations $\left(W\left(1^{-}, 90^{\circ}, 0^{\circ}\right), W\left(1^{+}, 90^{\circ}, 90^{\circ}\right), W\left(2^{+}, 90^{\circ}, 90^{\circ}\right)\right.$, and $\left.W\left(2^{+}, 135^{\circ}, 0^{\circ}\right)=0\right)$. The expected pattern for the occurrence of a ground-state decay in detectors at the angles $\theta$ and $\phi$ for spin and parity combination that can be excited with real photons is outlined in Table 1. For certain angle combinations, the vanishing angular distribution allows the observation of a pronounced spin and parity dependent analysing power $\Sigma$, e.g.,: 


$$
\begin{aligned}
\Sigma & =\frac{W\left(90^{\circ}, 0^{\circ}\right)-W\left(90^{\circ}, 90^{\circ}\right)}{W\left(90^{\circ}, 0^{\circ}\right)+W\left(90^{\circ}, 90^{\circ}\right)} \\
& =\left\{\begin{array}{l}
+1 \text { for } \pi=+ \\
-1 \text { for } \pi=-
\end{array}\right.
\end{aligned}
$$

or

$$
\begin{aligned}
\Sigma & =\frac{W\left(90^{\circ}, 0^{\circ}\right)-W\left(135^{\circ}, 0^{\circ}\right)}{W\left(90^{\circ}, 0^{\circ}\right)+W\left(135^{\circ}, 0^{\circ}\right)} \\
& =\left\{\begin{array}{c}
1 \text { for } J=2^{+} \\
0 \text { for } J^{\pi}=1^{+} \\
-1 \text { for } J^{\pi}=1^{-} .
\end{array}\right.
\end{aligned}
$$

The observed experimental asymmetry ratio $A$ can be defined as:

$$
\begin{aligned}
A_{\left(\theta_{1}, \phi_{1}\right) ;\left(\theta_{2}, \phi_{2}\right)} & =Q \times P \times \Sigma \\
& =\frac{N\left(\theta_{1}, \phi_{1}\right)-N\left(\theta_{2}, \phi_{2}\right)}{N\left(\theta_{1}, \phi_{1}\right)+N\left(\theta_{2}, \phi_{2}\right)},
\end{aligned}
$$

where $Q$ is the polarisation sensitivity of the setup and $P$ is the degree of polarisation in the entrance channel. The present measurements used fully linearly-polarised photon beams and, consequently, $P=1$. $Q$ includes the finite opening angles of the detectors, which diminishes the ideal ratio of the analysing power $\Sigma . N\left(\theta_{i}, \phi_{i}\right)$ are the relative-efficiency and electronic dead-time corrected count rates in the detector at angles $\theta_{i}$ and $\phi_{i}$.

The experiment employed a cylindrical (height $1.5 \mathrm{~cm}$, diameter $1.65 \mathrm{~cm}$ ) sample (mass: $7.6153 \mathrm{~g}$ ) of natural silicon, consisting of $92.2 \%{ }^{28} \mathrm{Si}, 4.7 \%{ }^{29} \mathrm{Si}$, and $3.1 \%{ }^{30} \mathrm{Si}$. The NRF setup used for this analysis was located $\approx 60 \mathrm{~m}$ downstream from the collision point. The detector array was the so-called $\gamma^{3}$ setup [16] consisting of four high-purity germanium (HPGe) detectors and four large-volume $\mathrm{LaBr}_{3}$ scintillation detectors. In this work, only the data from the HPGe detectors were used. Their positioning, relative to the momentum vector $(\theta$ angle) and polarisation vector of the electric field ( $\phi$ angle), is given in Table 2. The relative $\gamma$ ray detection efficiency was simulated using the GEANT4 toolkit [22] for each HPGe detector and fitted to the wellknown lines of a ${ }^{56} \mathrm{Co}$ source [23]. This procedure allows

Table 1 Expected peak occurrence (+), for a given spin and parity combination, $J^{\pi}$, in an even-even nucleus, in the spectrum recorded in a point-like detector placed under the angles $\theta$ and $\phi$ according to Eq. 1
Table $2 \theta$ and $\phi$ angle position of the HPGe detectors used in the present analysis. The $\theta$ angle is defined relative to the momentum direction of the incident photon beam and the $\phi$ angle relative to the (electric) polarisation vector of the incident fully linearly-polarised photon beam

\begin{tabular}{lll}
\hline Detector no. & $\theta$ & $\phi$ \\
\hline 1 & $90^{\circ}$ & $180^{\circ}$ \\
2 & $90^{\circ}$ & $90^{\circ}$ \\
3 & $90^{\circ}$ & $0^{\circ}$ \\
4 & $135^{\circ}$ & $180^{\circ}$ \\
\hline
\end{tabular}

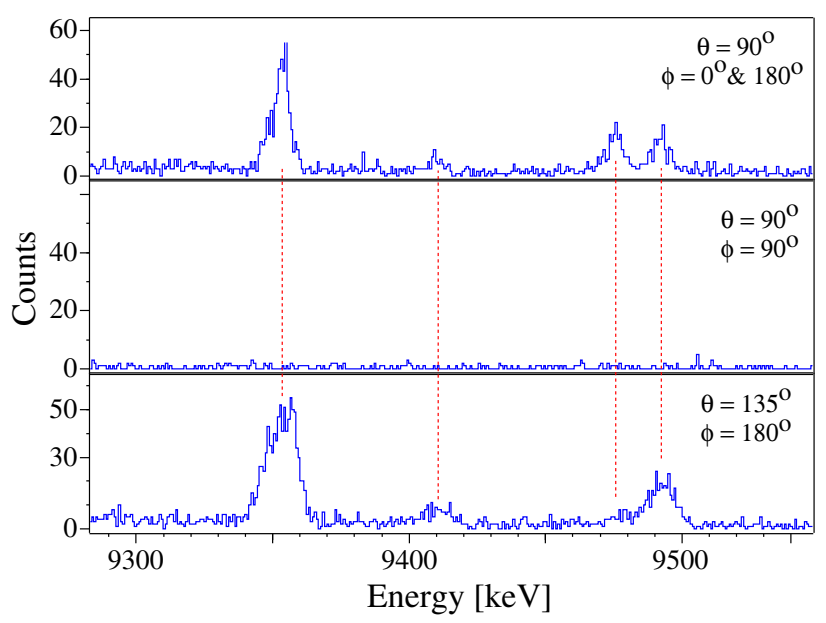

Fig. 1 Portions of $\gamma$-ray spectra from the ${ }^{\text {nat }} \operatorname{Si}\left(\gamma, \gamma^{\prime}\right)$ reaction recorded using the HPGe detectors of the $\gamma^{3}$ setup [16]. The angles at which the HPGe detectors were positioned are given in the respective panels. A definition of the angles is provided in the caption of Table 2. The mean energy of the incident photons was $9.4 \mathrm{MeV}$. The vertical (red) lines serve to guide the eye. For a discussion, see the text

cross normalisation of the relative efficiency of one detector to another. For each detector, a two-point energy calibration was carried out using the peaks of the $2614.8-\mathrm{keV}$ ${ }^{208} \mathrm{~Pb}$ background and the well-known energy of the groundstate decay $\left(E_{\gamma}=11441 \mathrm{keV}\right)$ from the 11446-keV level in ${ }^{28} \mathrm{Si}$. In order to enhance the statistics and decrease the statistical uncertainty, the spectra recorded in the detectors under the polar angle $\theta=90^{\circ}$ and azimuthal angles $\phi=0^{\circ}$ and $180^{\circ}$ were added and are in the following denoted as $(\theta, \phi)=(90,0)$. As the angular distributions are identical for those angles, this treatment is well justified.

Due to the high polarisation sensitivity of the setup and spin-selectivity of the reaction, the spin-parity assignment for levels which exhibit a ground-state transition is unambiguous. Examples of spectra recorded in the HPGe detectors of the $\gamma^{3}$ setup are presented in Figs. 1 and 2. The mean energies of the incident photons were $9.4 \mathrm{MeV}$ (Fig. 1) and $9.8 \mathrm{MeV}$ (Fig. 2), respectively.

Figures 1 and 2 show portions of the $\gamma$-ray spectra in which the ground-state decays of the excited states are present. The 


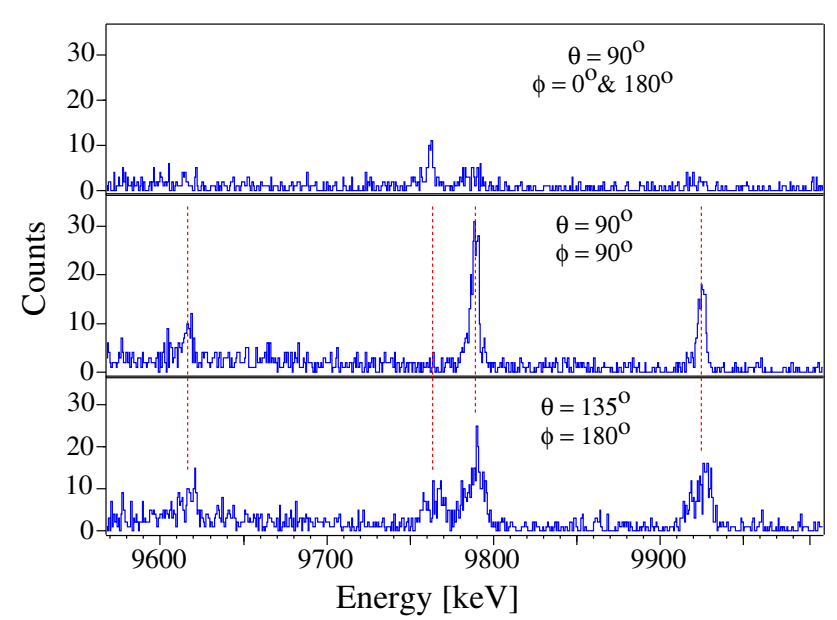

Fig. 2 Portions of $\gamma$-ray spectra from the ${ }^{n a t} \operatorname{Si}\left(\gamma, \gamma^{\prime}\right)$ reaction recorded using the HPGe detectors of the $\gamma^{3}$ setup. The angles at which the HPGe detectors were positioned are given in the respective panels. A definition of the angles is given in the caption of Table 2. The mean energy of the incident photons was $9.8 \mathrm{MeV}$. The vertical (red) lines serve to guide the eye. For a discussion, see the text

Table 3 Experimental results for a level tentatively attributed to ${ }^{29} \mathrm{Si}$ [9]. Given are the level energy, $E_{x}$, the observed $\gamma$-ray energy, $E_{\gamma}$, the possible spin and parity, $J^{\pi}$, the spin and parity, $J_{\text {lit }}^{\pi}$, from Ref. [24], and the observed asymmetry, $A_{(90,0) ;(135,0)}$. For a discussion, see the text

\begin{tabular}{lllll}
\hline$E_{x}[\mathrm{keV}]$ & $E_{\gamma}[\mathrm{keV}]$ & $J^{\pi}$ & $J_{\mathrm{lit}}^{\pi}$ & $A_{(90,0) ;(135,0)}$ \\
\hline $9412(3)$ & $9410(3)$ & $(1 / 2,3 / 2)^{+}$ & $3 / 2^{-}$ & $0.05(21)$ \\
\hline
\end{tabular}

observed levels exhibit the expected patterns as outlined in Table 1. For example, in Fig. 1, the levels corresponding to the peaks at $9354 \mathrm{keV}$ and $9496 \mathrm{keV}$ have $J^{\pi}=1^{+}$, while the level corresponding to the $9479 \mathrm{keV}$ peak has $J^{\pi}=2^{+}$, and no $1^{-}$level is present in the energy region covered by the incident photons. However, in Fig. 2, the levels at 9620, 9792 , and $9929 \mathrm{keV}$ are unambigously identified as $1^{-}$levels, while for the level at $9766 \mathrm{keV} J^{\pi}=1^{+}$can firmly be assigned. The levels at 9354, 9620, 9766, and $9792 \mathrm{keV}$ correspond to known spin-1 levels in ${ }^{30} \mathrm{Si}[10]$, a nucleus of low content in the sample.

In addition, a ground-state transition from a level at $9413 \mathrm{keV}$ is present in the spectra of Fig. 1. In the literature for ${ }^{29} \mathrm{Si}$ [9], a level with a sole ground-state $\gamma$-ray decay is reported at this energy. Interestingly, the neutronseparation threshold for this nucleus is $S_{n}=8473.6 \mathrm{keV}$, well below this level energy. In Ref. [24], a level at $9416 \mathrm{keV}$ with $J^{\pi}=3 / 2^{-}$is reported to be excited in the ${ }^{28} \mathrm{Si}(n, \gamma)$ reaction, with subsequent decay to the ground state. However, for a dipole excited level in an odd-mass nucleus, a pronounced angular correlation should also be present, but to a smaller extent than in an even-even one (see, e.g., Ref. [25]). For a $1 / 2^{+} \rightarrow 1 / 2^{\pi} \rightarrow 1 / 2^{+}$cascade, an isotropic distribution is expected, which is clearly ruled out by the non-observation of the $9410-\mathrm{keV}$ peak in the spectrum recorded at angles $\theta=90^{\circ}, \phi=90^{\circ}$ (see Fig. 1). For a $1 / 2^{+} \rightarrow 3 / 2^{\pi} \rightarrow 1 / 2^{+}$ cascade, a parity-dependent angular distribution is expected that would still result in the observation of at least a few
Table 4 Experimental results for levels attributed to ${ }^{28} \mathrm{Si}$, namely the level energy, $E_{x}$, the measured $\gamma$-ray energy, $E_{\gamma}$, the assigned spin and parity $J^{\pi}$, the spin and parity, $J_{\text {lit }}^{\pi}$, as found in the NNDC database [8], and the observed asymmetry,

$A_{\left(\theta_{1}, \phi_{1}\right) ;\left(\theta_{2}, \phi_{2}\right)}$ as defined in Eq. (4). If no peak was observed in one of the spectra, no asymmetries involving this detector are given

\begin{tabular}{|c|c|c|c|c|c|c|}
\hline$E_{x}[\mathrm{keV}]$ & $E_{\gamma}[\mathrm{keV}]$ & $J^{\pi}$ & $J_{\text {lit }}^{\pi}$ & $A_{(90,0) ;(135,0)}$ & $A_{(90,90) ;(135,0)}$ & $A_{(90,0) ;(90,90)}$ \\
\hline $9381(2)^{a}$ & 7601(2) & $b_{2}+$ & $2^{+}$ & $0.21(25)$ & & \\
\hline $9478(3)$ & $9476(3)$ & $2^{+}$ & $\left(2^{+}\right)$ & $0.84(16)$ & & \\
\hline $9494(3)$ & $9492(3)$ & $1^{+}$ & $\left(1^{+}\right)$ & $0.12(10)$ & & \\
\hline $9928(3)$ & $9926(3)$ & $1^{-}$ & $1^{-}$ & $-0.52(9)$ & $-0.02(10)$ & $-0.53(16)$ \\
\hline \multirow[t]{3}{*}{$10595(2)$} & 10593(3) & $1^{+}$ & $\left(1^{+}\right)$ & $0.13(5)$ & & \\
\hline & $8814(2)$ & & & $0.23(30)$ & & \\
\hline & $5615(2)$ & & & $0.09(17)$ & & \\
\hline 10722(3) & $10720(3)$ & $1^{+}$ & $\left(1^{+}\right)$ & $0.12(6)$ & & \\
\hline \multirow[t]{3}{*}{$10899(2)$} & 10896(3) & $1^{+}$ & $\left(1^{+}\right)$ & $0.13(4)$ & & \\
\hline & $9119(3)$ & & & $0.10(7)$ & $-0.67(5)$ & $0.72(5)$ \\
\hline & $5919(2)$ & & & $0.22(20)$ & & \\
\hline $10950(4)^{a}$ & $9169(4)$ & $(1,2)$ & 1 to 4 & $-0.06(40)$ & & \\
\hline $10992(3)^{c}$ & 10990(3) & $1^{-}$ & $\left(1,2^{+}\right)$ & & $0.02(22)$ & \\
\hline 11294(3) & 11292(3) & $1^{-}$ & $\left(1^{-}\right)$ & & $-0.18(37)$ & \\
\hline $11431(3)^{a}$ & $9650(3)$ & $\left(1,2^{+}\right)$ & $\left(2^{+}\right)$ & $0.28(19)$ & $-0.69(15)$ & $0.81(10)$ \\
\hline $11446.00(16)^{b}$ & $11441.0^{d}$ & $1^{+}$ & $\left(1^{+}\right)$ & $0.16(2)$ & $-0.98(1)$ & $0.99(1)$ \\
\hline
\end{tabular}

${ }^{a}$ Using the energy (1779 keV) of the $2_{1}^{+}$level from Ref. [8]

${ }^{b}$ Taken from Ref. [8].

${ }^{c}$ 9212-keV $\gamma$ ray from Ref. [8] not present, 10990(3) $\gamma$ ray known from [26].

${ }^{d}$ Taken from Ref. [8] and used for energy calibration 
Table 5 Experimental results for levels attributed to ${ }^{30} \mathrm{Si}$, namely the level energy, $E_{x}$, the measured $\gamma$-ray energy, $E_{\gamma}$, the assigned spin and parity, $J^{\pi}$, the spin and parity, $J_{\text {lit }}^{\pi}$, as found in the NNDC database [10], and the observed asymmetry, $A_{\left(\theta_{1}, \phi_{1}\right) ;\left(\theta_{2}, \phi_{2}\right)}$ as defined in Eq. (4). If no peak was observed in one of the spectra, no asymmetries involving this detector are given

\begin{tabular}{|c|c|c|c|c|c|c|}
\hline$E_{x}[\mathrm{keV}]$ & $E_{\gamma}[\mathrm{keV}]$ & $J^{\pi}$ & $J_{\text {lit }}^{\pi}$ & $A_{(90,0) ;(135,0)}$ & $A_{(90,90) ;(135,0)}$ & $A_{(90,0) ;(90,90)}$ \\
\hline $9356(3)$ & $9354(3)$ & $1^{+}$ & $\left(1,2^{+}\right)$ & $0.13(6)$ & & \\
\hline $9619(3)$ & $9617(3)$ & $1^{-}$ & $\left(1^{-}\right)$ & & $-0.11(22)$ & \\
\hline $9765(3)$ & $9763(3)$ & $1^{+}$ & $\left(1,2^{+}\right)$ & $0.26(19)$ & & \\
\hline 9791(3) & $9789(3)$ & $1^{-}$ & $\left(1^{-}\right)$ & $-0.4(1)$ & $0.14(10)$ & $-0.51(13)$ \\
\hline $10200(3)$ & 10198(3) & $1^{-}$ & $\left(1^{-}\right)$ & & $-0.34(20)$ & \\
\hline
\end{tabular}

events in the spectrum of the $\theta=90^{\circ}, \phi=90^{\circ}$ detector, even when considering the reduced $\gamma$-ray detection efficiency of the setup in this direction. Given the relatively small amount of ${ }^{29} \mathrm{Si}$ in the target, the $E 2$-excited $1 / 2^{+} \rightarrow 5 / 2^{+} \rightarrow 1 / 2^{+}$ cascade can be ruled out with a high degree of confidence. In fact, the observed asymmetry $A_{(90,0) ;(135,0)}$ measured by the two detectors in the polarisation plane $\phi=0^{\circ}$, indicates $J^{\pi}=1^{+}$, this making an assignment to an even-even nucleus more likely. Consequently, this level is only tentatively assigned to the $9413-\mathrm{keV}$ level reported in the literature for ${ }^{29} \mathrm{Si}$. In order to fully answer the question about which nucleus this level belongs to, a measurement with a target of different isotopic composition is mandatory.

The extracted experimental asymmetries are presented in Table 4 for levels assigned to ${ }^{28} \mathrm{Si}$, in Table 3 for the level that is tentatively assigned to ${ }^{29} \mathrm{Si}$, and in Table 5 for levels assigned to ${ }^{30} \mathrm{Si}$. The extracted values, for levels for which ground-state transitions were observed in both relevant detectors, are compared to the theoretical expectations for an eveneven nucleus in Fig. 3. Part (a) shows the $A_{(90,0) ;(90,90)}$ values, $A_{(90,90) ;(135,0)}$ values are shown in part (b), and part (c) the $A_{(90,0) ;(135,0)}$ recorded in the polarisation plane are plotted. From the plot the enhanced polarisation sensitivity for $M 1$-excited levels and reduced polarisation sensitivity for $E 1$-excited levels are obvious. This is because the detectors at $\phi=0^{\circ}$ and $\phi=180^{\circ}$ were positioned closer to the scattering target than the one at $\phi=90^{\circ}$, resulting in comparably large opening angles. Thus, the polarisation sensitivity for $J^{\pi}=1^{-}$levels is reduced to $Q \approx 0.5$ (part (a) and (c) in Fig. 3). At first the assignment for the level at $10200 \mathrm{keV}$ seems dubious. However, considering the reduction of the experimental asymmetry due to the detector opening angles, which can be extracted using the other $1^{-}$levels, within two sigma the parity of this level can be assigned to be negative. Hence, the observed data allow firm parity assignments for all $J=1$ levels.

For two levels in ${ }^{28} \mathrm{Si}$ (see Table 6) transitions to lowerlying excited states were observed. For the decays from these $1^{+}$levels to the first excited $2_{1}^{+}$level, no multipole-mixing ratio is known, only their relative-efficiency corrected relative intensities, $I_{\gamma}$, multiplied by the angular distribution,

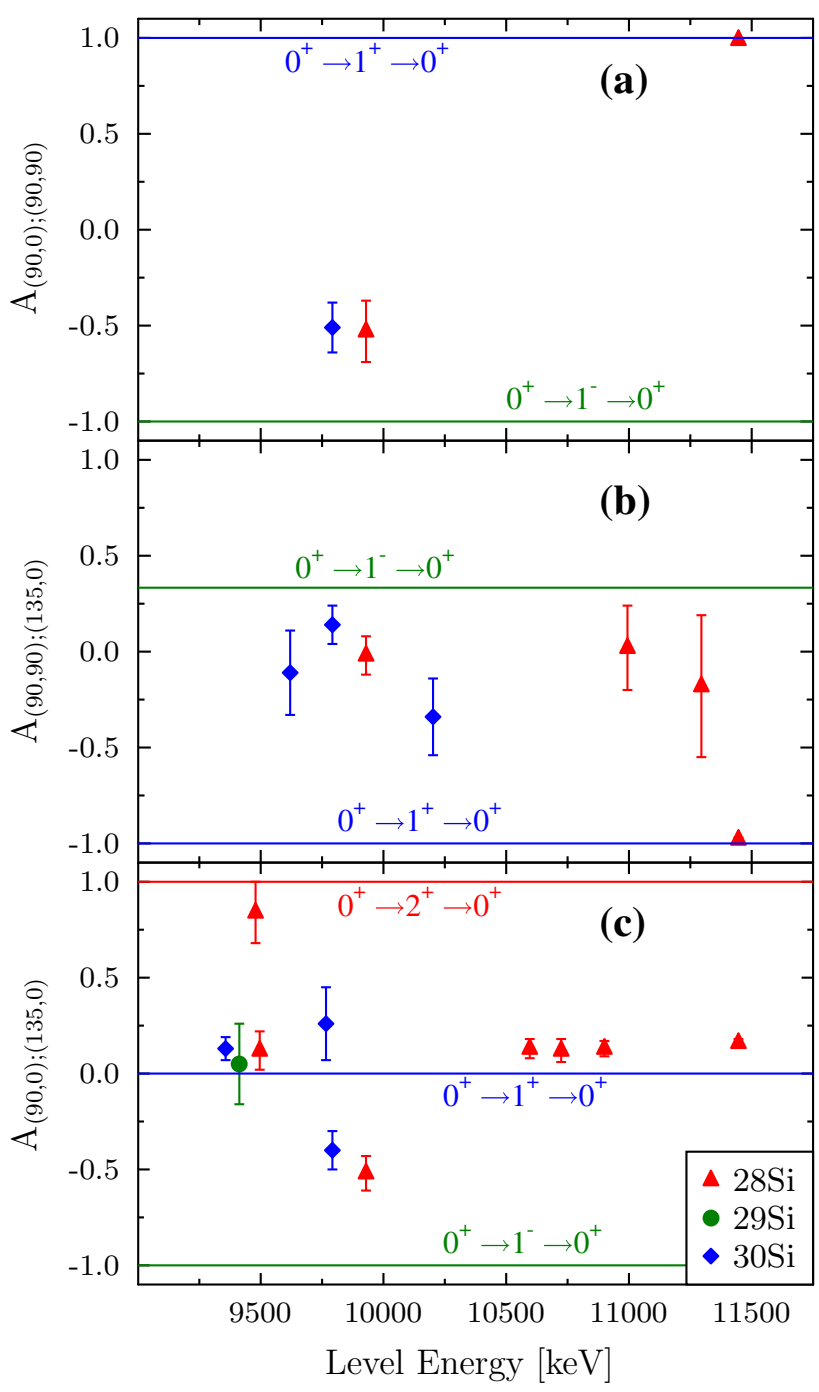

Fig. 3 Experimental asymmetries $A_{\left(\theta_{1}, \phi_{1}\right) ;\left(\theta_{2}, \phi_{2}\right)}$ for the observed ground-state decays as extracted from the spectra (see Tables 3, 4, and 5). The horizontal lines give the expected values, for an even-even nucleus, for a setup with perfect polarisation sensitivity, $Q=1$

$W(\theta, \phi)$, are given. For the $10596-\mathrm{keV}$ level, two known low-energy $\gamma$ rays are not observed, very likely due to the enhanced background at the energy of interest. One $\gamma$ ray linking this level to the first excited $2_{1}^{+}$level is observed here 
Table 6 Levels of ${ }^{28} \mathrm{Si}$ for which more than one depopulating $\gamma$ ray has been observed. As no multipole mixing ratio is known for the decay to the $2_{1}^{+}$level at $1779 \mathrm{keV}$, the product of the observed relative intensity,
$I_{\gamma, \text { rel }}$, normalised to the strongest transition and the angular distribution, $W(\theta, \phi)$, is presented

\begin{tabular}{llllll}
\hline$E_{x}[\mathrm{keV}]$ & $E_{\gamma}[\mathrm{keV}]$ & $J_{i}^{\pi}$ & $J_{f}^{\pi}$ & $I_{\gamma,(90,0)} \cdot W\left(90^{\circ}, 0^{\circ}\right)[\%]$ & $I_{\gamma,(135,0) \cdot W\left(135^{\circ}, 0^{\circ}\right)[\%]}$ \\
\hline $10595(2)$ & $10593(3)$ & $1^{+}$ & $0_{\mathrm{gs}}^{+}$ & $100(7)$ & $100(5)$ \\
& $8814(2)$ & & $2_{1}^{+}$ & $7(3)$ & $6(2)$ \\
$10899(2)$ & $5615(2)$ & & $0_{2}^{+}$ & $22(6)$ & $24(4)$ \\
& $10896(3)$ & $1^{+}$ & $0_{\mathrm{gs}}^{+}$ & $100(6)$ & $39(4)$ \\
& $9119(3)$ & & $2_{1}^{+}$ & $37(5)$ & $8(2)$ \\
\hline
\end{tabular}

for the first time. For the 10899-keV level, the decay to the first excited $0_{2}^{+}$level was not observed previously.

Interestingly, for the $10992-\mathrm{keV}$ level in ${ }^{28} \mathrm{Si}$, there was previously only a decay to the $2_{1}^{+}$level given in the database [8] but no ground-state decay. However, in the present work, only the ground-state decay is observed with no decay to the $2_{1}^{+}$level. A recent ${ }^{24} \operatorname{Mg}(\alpha, \gamma)$ experiment [26] established a dominant ground-state branch of $85(8) \%$ compared to a $15(3) \%$ branch to the first excited $2_{1}^{+}$level. As this level is comparatively weakly excited in the present experiment, it is likely that the decay to the $2_{1}^{+}$level is hidden in the background.

These data involve only a limited range of excitation energies. Nevertheless, the importance of the silicon isotopes, as outlined in the introduction, demands a more thorough and systematic investigation to establish their complete $M 1$ and $E 1$ responses below the neutron separation threshold and, possibly, even above. This work provides, at least for the levels in the energy range covered, the parity information.

\section{Summary}

Unambiguous spin and parity assignments are provided for levels in ${ }^{28} \mathrm{Si}$ and ${ }^{30} \mathrm{Si}$. Additionally, spins and parities of these levels established through this work allow the use of natural silicon samples as standard to calibrate angular distribution measurements similar to these carried out here.

Acknowledgements The authors are indebted to the accelerator crew of $\mathrm{HI} \gamma \mathrm{S}$, who provided excellent experimental conditions. The UWS group acknowledges financial support by the UK Science and Technology Facilities Council (UK-STFC). The TU Darmstadt members acknowledge financial support by the Deutsche Forschungsgemeinschaft (SFB-1245). In addition, OP and JK acknowledge financial support by the German state of Hesse under the grant Nuclear Photonics within the LOEWE program. The Cologne group acknowledges financial support from the German Bundesministerium für Bildung und Forschung under Grant 05P2018/ELI-NP. This work was supported by the US Department of Energy (DOE), Office of Nuclear Physics, under Grant no. DE-FG02-97ER41033 (Duke University) and DE-FG0297ER41041 (UNC) and the U.S. National Science Foundation under
Grants nos. PHY-1565546 (NSCL) and PHY-1913028. GB and DAJ acknowledge support of the UK EPSRC (Grant no. EP/N028694/1), the EC's Laserlab-Europe, H2020 EC-GA 654148 and ELI - Extreme Light Infrastructure phase 2 from the European Regional Development Fund.

Data Availability Statement This manuscript has no associated data or the data will not be deposited. [Authors' comment: The collaboration will provide the raw (unprocessed data files), processed spectra, and meta data upon request by a third party.]

Open Access This article is licensed under a Creative Commons Attribution 4.0 International License, which permits use, sharing, adaptation, distribution and reproduction in any medium or format, as long as you give appropriate credit to the original author(s) and the source, provide a link to the Creative Commons licence, and indicate if changes were made. The images or other third party material in this article are included in the article's Creative Commons licence, unless indicated otherwise in a credit line to the material. If material is not included in the article's Creative Commons licence and your intended use is not permitted by statutory regulation or exceeds the permitted use, you will need to obtain permission directly from the copyright holder. To view a copy of this licence, visit http://creativecomm ons.org/licenses/by/4.0/.

\section{References}

1. S. Reddy, M. Prakash, J.M. Latimer, Phys. Rev. D 58, 013009 (1998)

2. A. Burrows, S. Reddy, T.A. Thomspon, Nucl. Phys. A 777, 356 (2006)

3. K.-H. Langanke, G. Martinez-Pinedo, P. von Neumann Cosel, A. Richter, Phys. Rev. Lett. 93, 202501 (2004)

4. A.A. Dziohev et al., Phys. Rev. C 94, 015805 (2016)

5. K. Heyde, P. von Neumann-Cosel, A. Richter, Rev. Mod. Phys. 82, $2365(2010)$

6. A.A. Dziohev, V.V. Vdovin, Acta. Phs. Pol B48, 667 (2017)

7. A.A. Dziohev, A.I. Vdovin, J. Wambach, V.Y. Ponomarev, Phys. Rev. C 89, 035805 (2014)

8. M. Shamsuzzoha Basunia, Nucl. Data. Sheets 114, 1189 (2013)

9. M. Shamsuzzoha Basunia, Nucl. Data. Sheets 113, 909 (2012)

10. M. Shamsuzzoha Basunia, Nucl. Data. Sheets 111, 2331 (2010)

11. R. Schneider et al., Nucl. Phys. A 323, 13 (1979)

12. H. Matsubara et al., Phys. Rev. Lett. 115, 102501 (2015)

13. U.E.P. Berg et al., Phys. Lett. 140B, 191 (1984)

14. U. Kneissl, H.H. Pitz, A. Zilges, Prog. Part. Nucl. Phys. 37, 349 (1996) 
15. J. Beller et al., Phys. Lett. B 741, 128 (2015)

16. B. Löher et al., Nucl. Inst. Meth. A723, 136 (2013)

17. H. Weller et al., Prog. Part. Nucl. Phys. 62, 257 (2009)

18. L.W. Fagg, S.S. Hanna, Rev. Mod. Phys. 31, 711 (1959)

19. K. Krane, R. Steffen, R. Wheeler, At. Data Nucl. Data Tables 11, $351(1973)$

20. C.A. Ur et al., Rom. Rep. Phys. 68, S483 (2016)

21. J. Isaak, PhD thesis, Gutenberg University Mainz, unpublished (https://publications.ub.uni-mainz.de/theses/volltexte/2016/ 100000664/pdf/100000664.pdf) (2019)
22. S. Agnostinelli et al., Nucl. Inst. Meth. B506, 250 (2003)

23. H. Junde, H. Su, Y. Dong, Nucl. Data. Sheets 112, 1513 (2011)

24. L.R. Medsker, H.E. Jackson, J.L. Yntema, Phys. Rev. C 9, 1851 (1974)

25. N. Pietralla et al., J. Phys. Conf. Series 205, 012036 (2010)

26. E. Strandberg et al., Phys. Rev. C 77, 055801 (2008) 